\title{
A Visual Axis Stability Control Algorithm for Airborne Photoelectric Tracking System
}

\author{
Zhen Wang ${ }^{1,}$, , Hongjie $\mathrm{Hu}^{2, \mathrm{~b}}$ \\ ${ }^{1}$ School of Automation Science, Beihang University, Beijing, 100191, China; \\ 2 School of Automation Science, Beihang University, Beijing, 100191, China. \\ awangzhen_sdu@163.com, bhhj@buaa.edu.cn
}

Keywords: Photoelectric tracking, Visual axis stability, Airplane disturbance compensation model, Visual axis space position observation model.

\begin{abstract}
The visual axis stabilization technology is a key technology for improving the tracking accuracy and image stability recognition of the photoelectric tracking system. In this paper, a compensation model for yaw and pitch two-axis disturbances is established to achieve space velocity compensation, based on the design of a tracking system for airborne electro-optical imaging equipment. A spatial position observation model with two degrees of freedom of yaw and pitch is established, through the coordinate transformation technique. The spatial position closed loop of the visual axis in pitch and yaw is achieved. Firstly, the overall structure of the system is given in the paper. Then the mathematical model of the visual axis disturbance compensation model and the visual axis position observation model are deduced. The system control block diagram is designed separately. The visual axis stability control algorithm is certificated on the SIMULINK simulation model.
\end{abstract}

\section{Introduction}

With the rapid development of high-precision servo control technology, high-precision servo turntables have been widely used in military weapons and other fields. The photoelectric tracking technology is a major technology which apply the servo turntable to the fighter's long-range detection imaging device platform. The servo turntable drives the imaging device to rotate within the inertial space for scanning long-distance targets, and quickly lock and track the target after detecting the target. The visual axis stabilization technology is a key technology to improve the tracking accuracy of photoelectric tracking systems and image recognition of photoelectric imaging devices. The visual axis stabilization technology is a vital technology to improve the tracking accuracy of photoelectric tracking systems and image recognition of photoelectric imaging devices $[1,2]$.

The airborne photoelectric tracking system designed in this paper is a three-axis turntable system, which consists of three axes: yaw, pitch, and derotation for achieving the movement of sight axis in two freedom degrees of yaw and pitch. The derotation turntable is a follow-up mechanism, which turning angle is half the sum of the yaw and the pitch motor turning angle. Its main function is to eliminate the image rotation of the photoelectric imaging device.

When there is a disturbance in the inertial space, the airborne photoelectric tracking system can decouple the disturbance to the two-axis turntable of yaw and pitch through the compensation model of the body disturbance and output the corresponding compensated turning angle through the yaw and pitch turntable. The closed-loop control system ensures the visual axis stability of the photoelectric tracking device and creates a stable condition for the processing and stitching of the image signals [3].

\section{Overall System Design}

The overall structure of the airborne optoelectronic tracking system is shown in Figure 1. The master computer sends the control instruction through the signal processing box and obtains the current control status and other information of the system from the signal processing box and provides 
it to the user, which is convenient for the user to handle and judge. The infrared camera acquires the image information of the dynamic target. Then the signal processing box analyzes and solves the image to obtain the miss distance of the target in two directions and sends the miss distance information of yaw and pitch to the servo control system.

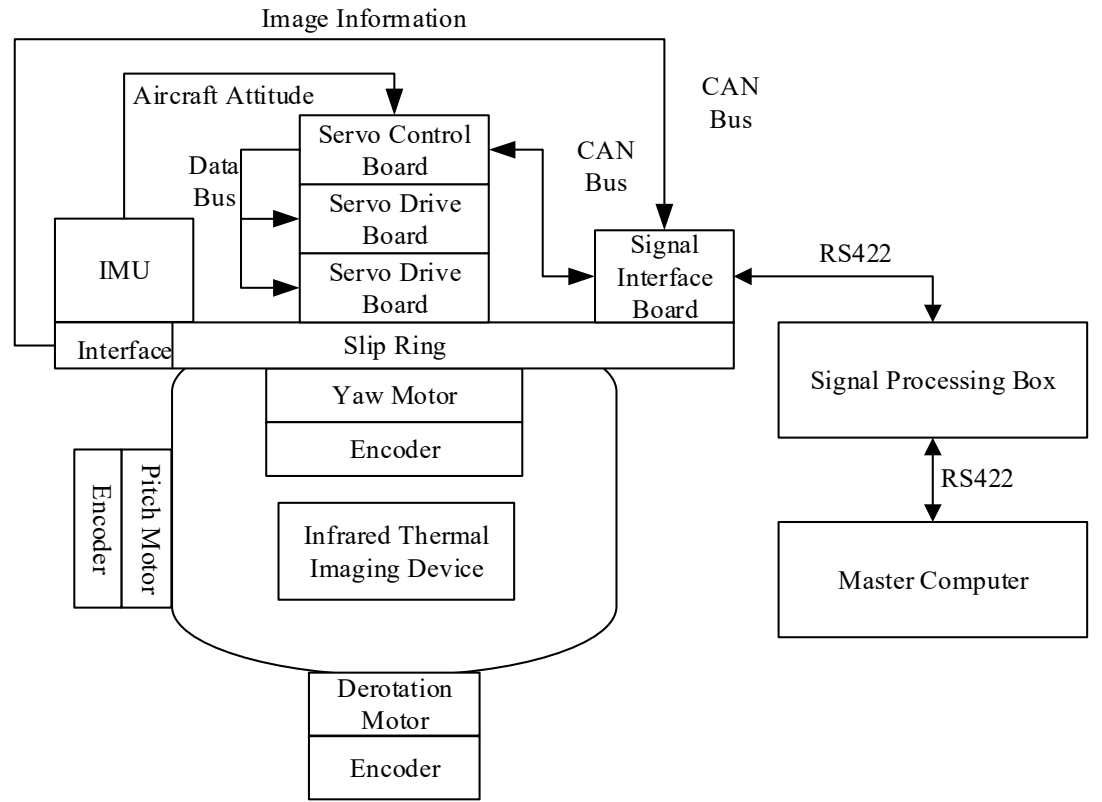

Fig. 1 Photoelectric Tracking System Overall Structure

Servo control system consists of one servo control board and two servo drive boards. After receiving the miss distance information, the servo control board completes the stable scanning and tracking algorithm construction according to aircraft disturbance attitude angle and angular velocity information measured by IMU (Inertial Measurement Unit) and gyroscopes, and the motor output position angle measured by the photoelectric encoder. The calculated control result is transmitted to two servo drive boards. The servo drive board drives the permanent magnet synchronous motor of the yaw, pitch, and derotation axis, to complete the scanning and trace and other operations. In addition, DSP and FPGA chip are the cores chip of the servo control system [4]. The servo control algorithms are all completed in servo control board. Airborne photoelectric tracking system control block diagram shown in Figure 2.

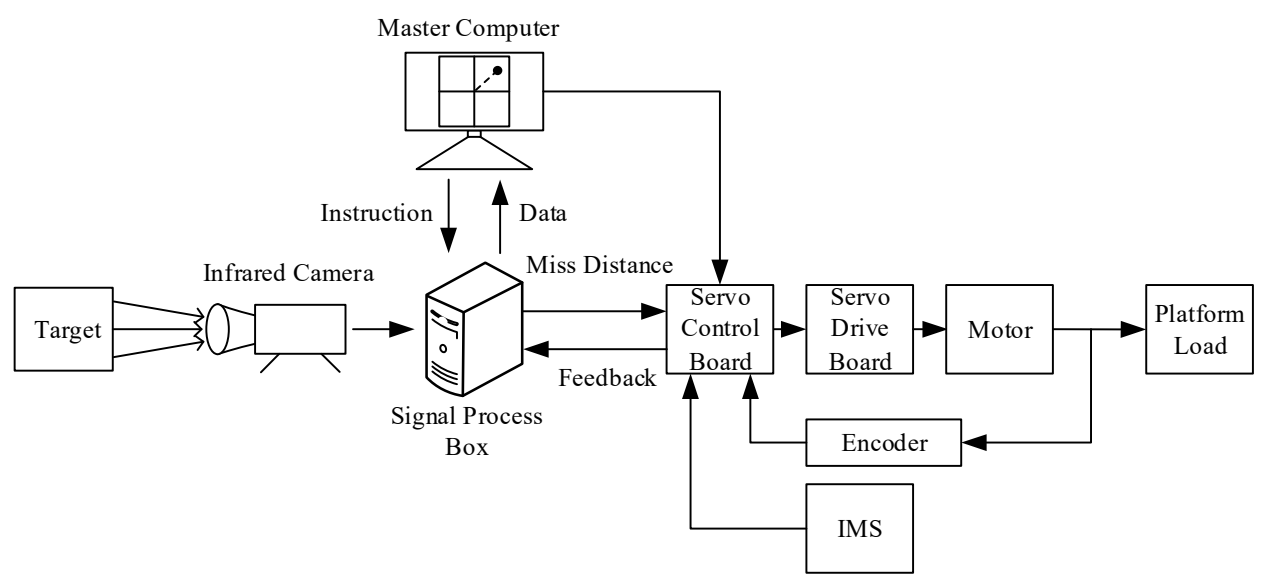

Fig. 2 Photoelectric Tracking System Control Block

\section{Airplane Disturbance Compensation Modeling and Simulation}

The principle of the airplane disturbance compensation model is to measure the three attitude angular velocities of the airplane in the geodetic coordinate system by the fiber optic gyroscope, and then mapped equivalently to the turntable coordinate system. Finally, the motor drives the two axis turrets respectively to realize disturbance compensation $[5,6]$. 
Next, we established the spatial coordinate systems of airplane. The specific definition is as follows: airplane coordinate system $O X_{d} Y_{d} Z_{d}, O Y_{d}$ axis is in the horizontal plane of the airplane, and coincides with the vertical axis, pointing to the airplane head is positive, $O X_{d}$ axis is located in the plane of the airplane, and is perpendicular to $O Y_{d}$ axis. $O Z_{d}$ axis is perpendicular to the airplane's level, which satisfies the right-handed coordinate system and is positive vertically. The yaw axis coordinate system $O X_{a} Y_{a} Z_{a}$ completely coincides with $O X_{d} Y_{d} Z_{d}$ in the initial state of the system. When the motor moves, except that the $O Z_{a}$ axis always coincides with the $O Z_{d}$ axis, the $O X_{a}$ and $O Y_{a}$ axes are always stationary with the yaw frame. The pitch axis coordinate system $O X_{f} Y_{f} Z_{f}$ is completely coincident with the $O X_{a} Y_{a} Z_{a}$ coordinate system in the initial state of the system. When the motor moves, the $O Y_{f}$ and $O Z_{f}$ axis are all relatively stationary with the pitch frame except that the $O X_{f}$ axis always coincides with the $O X_{a}$ axis. The infrared camera imaging device is mounted on the pitch axis, which normal line is parallel to the $O Y_{f}$ axis. Then, the pitch motor rotation angle $\theta_{f}$ is the rotation angle of the $O X_{f} Y_{f} Z_{f}$ coordinate system relative to the $O X_{a} Y_{a} Z_{a}$ coordinate system. Similarly, the yaw motor rotation angle $\theta_{a}$ is the rotation angle of the $O X_{a} Y_{a} Z_{a}$ coordinate system relative to the $O X_{d} Y_{d} Z_{d}$ coordinate system. At the same time, the visual axis of the infrared camera is uniquely determined by $\theta_{a}$ and $\theta_{f}$.

When the airplane has an angular velocity disturbance $\omega_{d}$ of relative inertial space, it is first coupled to the yaw coordinate system as the constraints of the platform frame. Because the $O X_{f}$ axis always coincides with the $O X_{a}$ axis, the disturbance is coupled into the pitch coordinate system. The relative positional relationship between the airplane coordinate system and the yaw coordinate system and the angular velocity relationship are shown in Figure 3.

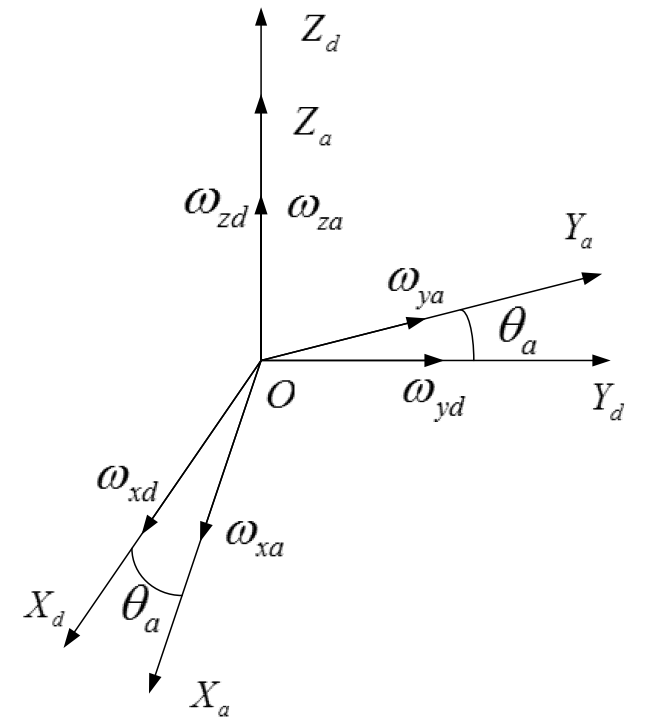

Fig. 3 Airplane and Yaw Coordinate System

$\omega_{x d}, \omega_{y d}, \omega_{z d}$ are the three-axis disturbance angular velocity in the airplane coordinate system measured by the fiber optic gyro. $\omega_{x a}, \omega_{y a}, \omega_{z a}$ are the angular velocities of the disturbance which transformed to the yaw coordinate system, $\dot{\theta}_{a}$ is the compensation angular velocity of the yaw motor output. According to the coordinate transformation relationship, there are:

$$
\left[\begin{array}{c}
\omega_{x a} \\
\omega_{y a} \\
\omega_{z a}
\end{array}\right]=\left[\begin{array}{ccc}
\cos \theta_{a} & \sin \theta_{a} & 0 \\
-\sin \theta_{a} & \cos \theta_{a} & 0 \\
0 & 0 & 1
\end{array}\right]\left[\begin{array}{c}
\omega_{x d} \\
\omega_{y d} \\
\omega_{z d}
\end{array}\right]+\left[\begin{array}{c}
0 \\
0 \\
\dot{\theta}_{a}
\end{array}\right]
$$

The relative positional relationship between the yaw and pitch coordinate system is shown in Figure 4. 


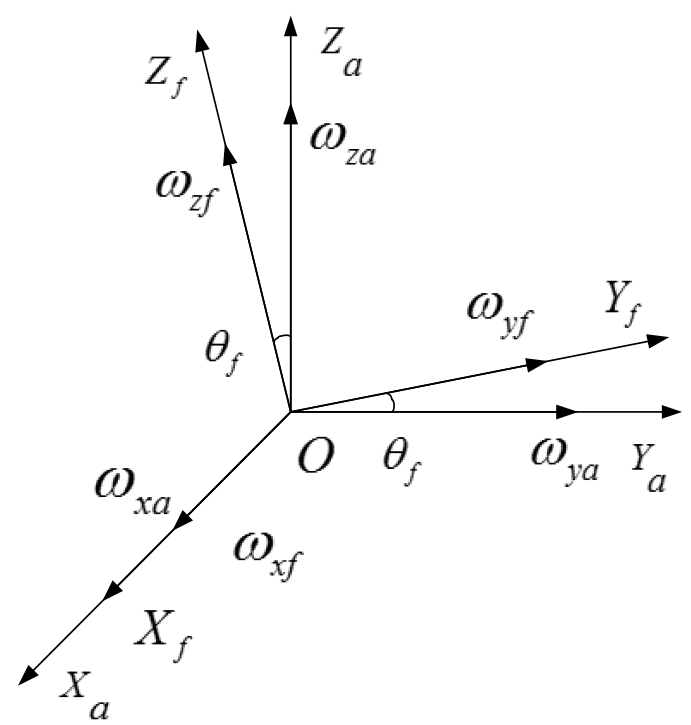

Fig. 4 Yaw and Pitch Coordinate System

$\omega_{x a}, \omega_{y a}, \omega_{z a}$ are the three-axis disturbance angular velocity in the yaw coordinate system. $\omega_{x f}$, $\omega_{y f}, \omega_{z f}$ are the three-axis disturbance angular velocity in the pitch coordinate system. $\dot{\theta}_{f}$ is the compensation angular velocity of the pitch motor output. According to the coordinate transformation relationship, there are:

$$
\left[\begin{array}{c}
\omega_{x f} \\
\omega_{y f} \\
\omega_{z f}
\end{array}\right]=\left[\begin{array}{ccc}
1 & 0 & 0 \\
0 & \cos \theta_{f} & \sin \theta_{f} \\
0 & -\sin \theta_{f} & \cos \theta_{f}
\end{array}\right]\left[\begin{array}{c}
\omega_{x a} \\
\omega_{y a} \\
\omega_{z a}
\end{array}\right]+\left[\begin{array}{c}
\dot{\theta}_{f} \\
0 \\
0
\end{array}\right]
$$

From the matrix formula (1) and (2), the relationship between the compensated angular velocity of the yaw motor and the pitch motor and the disturbance angular velocity is as follows:

$$
\left[\begin{array}{c}
\omega_{x f} \\
\omega_{y f} \\
\omega_{z f}
\end{array}\right]=\left[\begin{array}{ccc}
1 & 0 & 0 \\
0 & \cos \theta_{f} & \sin \theta_{f} \\
0 & -\sin \theta_{f} & \cos \theta_{f}
\end{array}\right]\left[\begin{array}{ccc}
\cos \theta_{a} & \sin \theta_{a} & 0 \\
-\sin \theta_{a} & \cos \theta_{a} & 0 \\
0 & 0 & 1
\end{array}\right]\left[\begin{array}{c}
\omega_{x d} \\
\omega_{y d} \\
\omega_{z d}
\end{array}\right]+\left[\begin{array}{c}
0 \\
\sin \theta_{f} \cdot \dot{\theta}_{a} \\
\cos \theta_{f} \cdot \dot{\theta}_{a}
\end{array}\right]\left[\begin{array}{c}
\dot{\theta}_{f} \\
0 \\
0
\end{array}\right]
$$

Finally, a compensation model of the airplane's disturbance angular velocity was established.

$$
\left\{\begin{array}{l}
\dot{\theta}_{a}=-\omega_{x d} \sin \theta_{a} \tan \theta_{f}+\omega_{y d} \cos \theta_{a} \tan \theta_{f}-\omega_{z d} \\
\dot{\theta}_{f}=-\omega_{x d} \cos \theta_{a}-\omega_{y d} \sin \theta_{a}
\end{array}\right.
$$

When the angular velocity output by the yaw motor and pitch motor satisfies the above conditions, the full compensation of the airplane attitude disturbance angular velocity can be achieved.

When the airborne electro-optical tracking system is in the tracking mode, the miss distance pixel information is sent to the servo control board through the signal interface board. The servo control board realizes the closed loop of the space velocity loop through the compensation model of the airplane disturbance, eliminates the influence of the airplane disturbance.

Now we use SIMULINK to simulate space target miss distance information captured by infrared thermal imaging equipment. Assume that the pointing direction of the yaw axis is $30^{\circ}$ and the pitch axis is $5^{\circ}$.At the same time, the airplane disturbance angular velocity is $\omega_{x d}=1^{\circ} \cos (2 \pi t), \omega_{y d}=$ $1^{\circ} \cos (2 \pi t), \omega_{z d}=10^{\circ} \cos (2 \pi 0.1 t)$. We get the following compensation motor speed and pitch motor compensation angular velocity simulation. The actual spatial angular velocity of the yaw axis is stabilized within $\pm 0.005^{\circ} / s$, and the actual spatial angular velocity of the pitch axis is stable within $\pm 0.007^{\circ} / s$. 

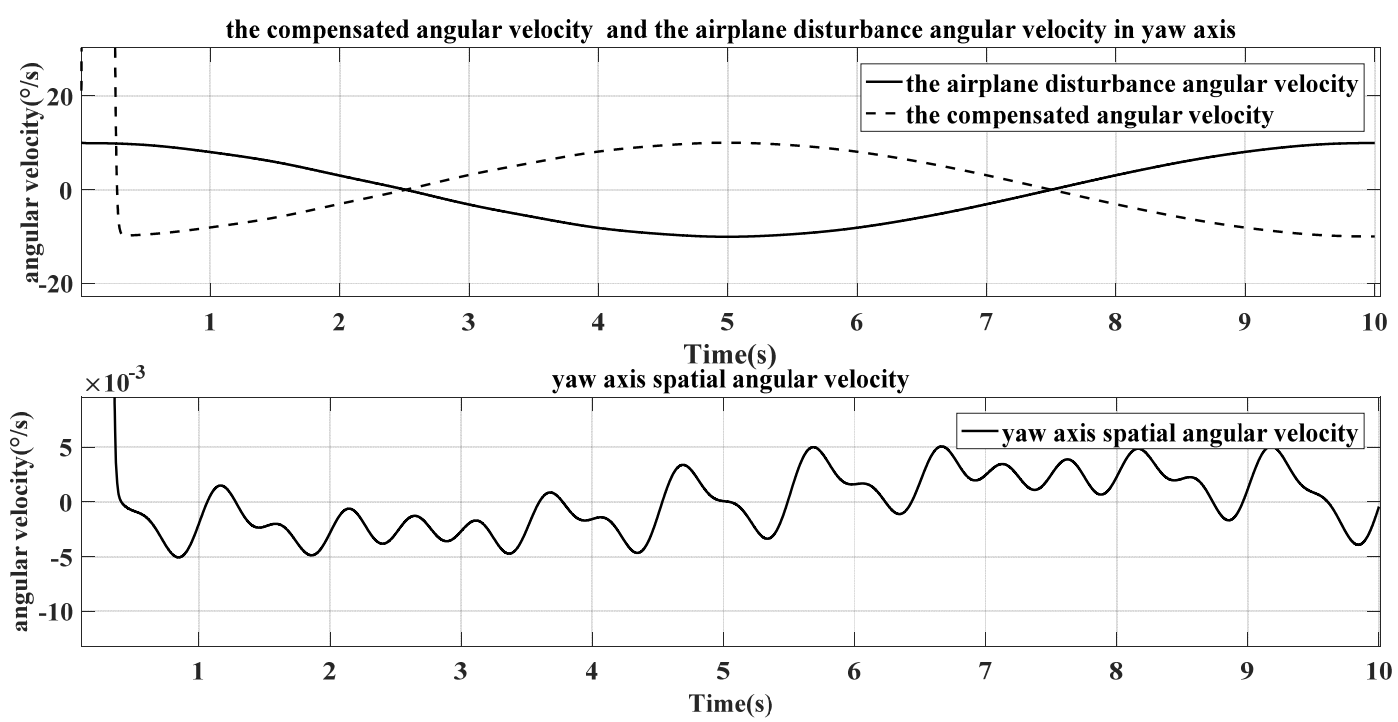

Fig. 5 Compensated Angular Velocity and Airplane Disturbance Angular Velocity in Yaw Axis
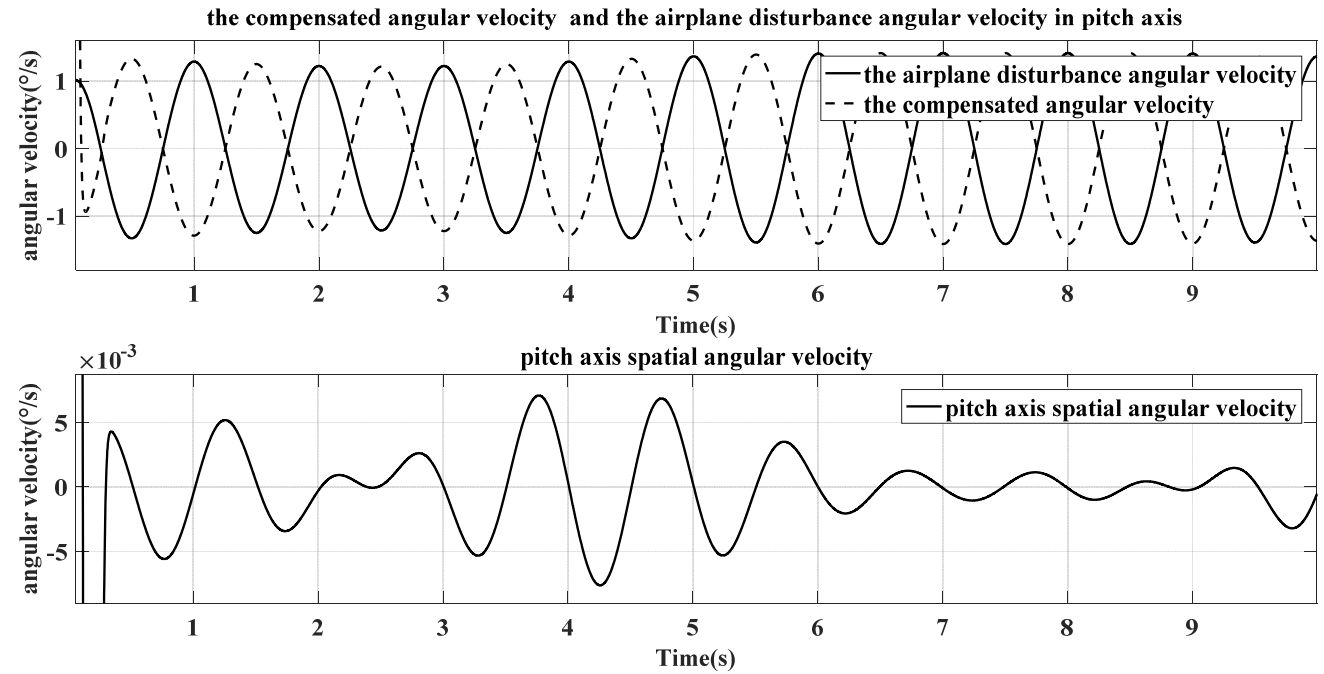

Fig. 6 Compensated Angular Velocity and Airplane Disturbance Angular Velocity in Pitch Axis

\section{Observational Models for Visual Axis Space Position Modeling and Simulation}

The principle of establishing the observation model of the visual axis space position is that, we firstly measured the attitude angles $K, P, R$ of the airplane in yaw, pitch, roll directions by IMU, then acquired the orientation of visual axis of the thermal imaging camera $\theta_{a}, \theta_{f}$ in airplane coordinate system by the encoder. Therefore, we observed the orientation of visual axis in the inertial space coordinate system $A_{1}, E_{1}$.

Subsequently, we establish the inertial coordinate system $O X Y Z$ and airplane coordinate system $O X_{d} Y_{d} Z_{d} . O Y_{d}$ axis is in the horizontal plane of the airplane, and coincides with the vertical axis, pointing to the airplane head is positive, $O X_{d}$ axis is located in the plane of the airplane, and is perpendicular to $O Y_{d}$ axis. $O Z_{d}$ axis is perpendicular to the airplane's level, which satisfies the righthanded coordinate system and is positive vertically. In the inertial coordinate system $O X Y Z, O Y$ axis points to the north, the $O X$ axis points to the south, and the $O Z$ axis points to the sky at the local horizontal. $O X Y Z$ completely coincides with $O X_{d} Y_{d} Z_{d}$ in the initial state of the system. $A_{1}$ is the angle between the projection of the visual axis of the infrared camera in $O X Y$ plane and $O Y$ axis, counterclockwise is positive. At the same time, $E_{1}$ is the angle between the visual axis of the imaging device of the infrared camera and the plane $O X Y$, and the upward direction is positive. $\theta_{a}$ is the angle between the projection of the visual axis of the infrared thermal camera on $O X_{d} Y_{d}$ plane and $O Y_{d}$, and is counterclockwise positive. $\theta_{f}$ is the angle between the visual axis and $O X_{d} Y_{d}$ plane. 
We assume that there is a point $M$ in $O X Y Z$ as the visual axis, the coordinate is $(x, y, z)$, and the distance is unit length 1 . Therefore, the inertial space coordinate and inertial space polar coordinate have the following conversion relationship:

$$
\left\{\begin{array}{l}
x=\cos E_{1} \sin A_{1} \\
y=\cos E_{1} \cos A_{1} \\
z=\sin E_{1}
\end{array}\right.
$$

Similarly, the coordinates of the $M$ point in $O X_{d} Y_{d} Z_{d}$ are $\left(x_{d}, y_{d}, z_{d}\right)$, and the conversion relations with airplane polar coordinates are as follows:

$$
\left\{\begin{array}{l}
x_{d}=\cos \theta_{f} \sin \theta_{a} \\
y_{d}=\cos \theta_{f} \cos \theta_{a} \\
z_{d}=\sin \theta_{f}
\end{array}\right.
$$

First, we assume that only when the roll angle changes by $R$, the airplane coordinate system is considered to rotate the $R$ angle counterclockwise around $Y_{d}$ axis, as shown in Figure 7:

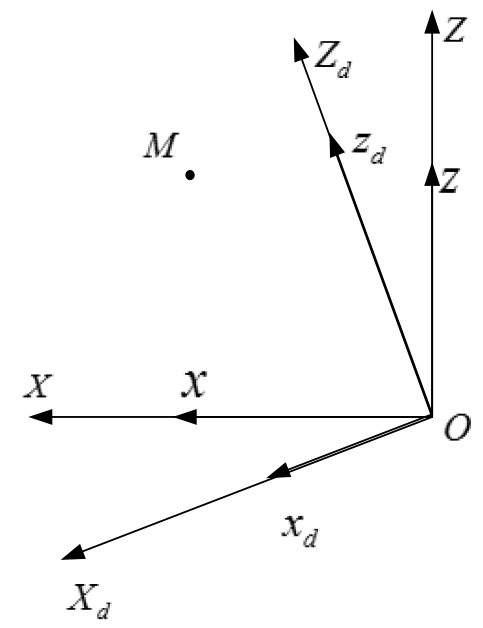

Fig. 7 Coordinate Transformation Relationship when Roll Angle Changes

We can get the coordinate transformation relationship when roll angle changes from Figure 7:

$$
\left[\begin{array}{l}
x_{d} \\
y_{d} \\
z_{d}
\end{array}\right]=\left[\begin{array}{ccc}
\cos R & 0 & \sin R \\
0 & 1 & 0 \\
-\sin R & 0 & \cos R
\end{array}\right]\left[\begin{array}{l}
x \\
y \\
z
\end{array}\right]
$$

In the same way, we can obtain that when the airplane coordinate system rotates the $P$ angle around $X_{d}$ axis, the coordinate transformation relationship is as follows:

$$
\left[\begin{array}{l}
x_{d} \\
y_{d} \\
z_{d}
\end{array}\right]=\left[\begin{array}{ccc}
1 & 0 & 0 \\
0 & \cos P & \sin P \\
0 & -\sin P & \cos P
\end{array}\right]\left[\begin{array}{l}
x \\
y \\
z
\end{array}\right]
$$

Then, we can obtain that when the airplane coordinate system rotates the $K$ angle around $Z_{d}$ axis, the coordinate transformation relationship is as follows:

$$
\left[\begin{array}{l}
x_{d} \\
y_{d} \\
z_{d}
\end{array}\right]=\left[\begin{array}{ccc}
\cos K & \sin K & 0 \\
-\sin K & \cos K & 0 \\
0 & 0 & 1
\end{array}\right]\left[\begin{array}{l}
x \\
y \\
z
\end{array}\right]
$$

Finally, the coordinate transformation equation of the coordinates of the inertial coordinate system to the airplane coordinate system is:

$$
\begin{gathered}
{\left[\begin{array}{l}
x_{d} \\
y_{d} \\
z_{d}
\end{array}\right]=T\left[\begin{array}{l}
x \\
y \\
z
\end{array}\right]} \\
T=\left[\begin{array}{ccc}
1 & 0 & 0 \\
0 & \cos P & \sin P \\
0 & -\sin P & \cos P
\end{array}\right]\left[\begin{array}{cccc}
\cos R & 0 & \sin R \\
0 & 1 & 0 \\
-\sin R & 0 & \cos R
\end{array}\right]\left[\begin{array}{ccc}
\cos K & \sin K & 0 \\
-\sin K & \cos K & 0 \\
0 & 0 & 1
\end{array}\right]
\end{gathered}
$$

From the matrix formulae (5) (6) (10), we can obtain the thermal camera visual axis directional relationship between the airplane coordinate system and the inertial space coordinate system: 


$$
\left[\begin{array}{c}
\cos \theta_{f} \sin \theta_{a} \\
\cos \theta_{f} \cos \theta_{a} \\
\sin \theta_{f}
\end{array}\right]=T\left[\begin{array}{c}
\cos E_{1} \sin A_{1} \\
\cos E_{1} \cos A_{1} \\
\sin E_{1}
\end{array}\right]
$$

Conversely, we know that the infrared camera pointing angles $\theta_{f}, \theta_{a}$ and the airplane attitude angles $K, P, R$, then we can observe the visual axis pointing angles of the infrared camera $A_{1}, E_{1}$ in the inertial space coordinate system:

$$
\left[\begin{array}{c}
\cos E_{1} \sin A_{1} \\
\cos E_{1} \cos A_{1} \\
\sin E_{1}
\end{array}\right]=T^{-1}\left[\begin{array}{c}
\cos \theta_{f} \sin \theta_{a} \\
\cos \theta_{f} \cos \theta_{a} \\
\sin \theta_{f}
\end{array}\right]
$$

Finally, we can observe the visual axis pointing angles of the infrared camera $A_{1}, E_{1}$ :

$$
\left\{\begin{array}{l}
A_{1}=\tan ^{-1} \frac{x}{y} \\
E_{1}=\sin ^{-1} z
\end{array}\right.
$$

Since the range of $\tan ^{-1}$ is $(-\pi / 2, \pi / 2)$, the correct value of $A_{1}$ needs to be judged based on the sign of $y$ :

$$
\begin{cases}A_{1}=\tan ^{-1} \frac{x}{y} & (y>0) \\ A_{1}=\pi+\tan ^{-1} \frac{x}{y} & (y<0)\end{cases}
$$

When airborne photoelectric tracking system is working on scanning mode, the master computer sends a scan command through the CAN bus and transmits it to the servo control board. The scanning instructions include the scanning range of the visual axis in yaw and pitch, the yaw forward and reverse scanning speed, the pitch forward and reverse scanning speed. We obtain the spatial orientation of the visual axis in the inertial coordinate system through the observation model, as a feedback of the spatial position loop. On the other hand, we collect the airplane's angular velocity through fiber optic gyro. After the motor output compensation, the spatial angular velocity is formed as the feedback of the space velocity loop[7,8].

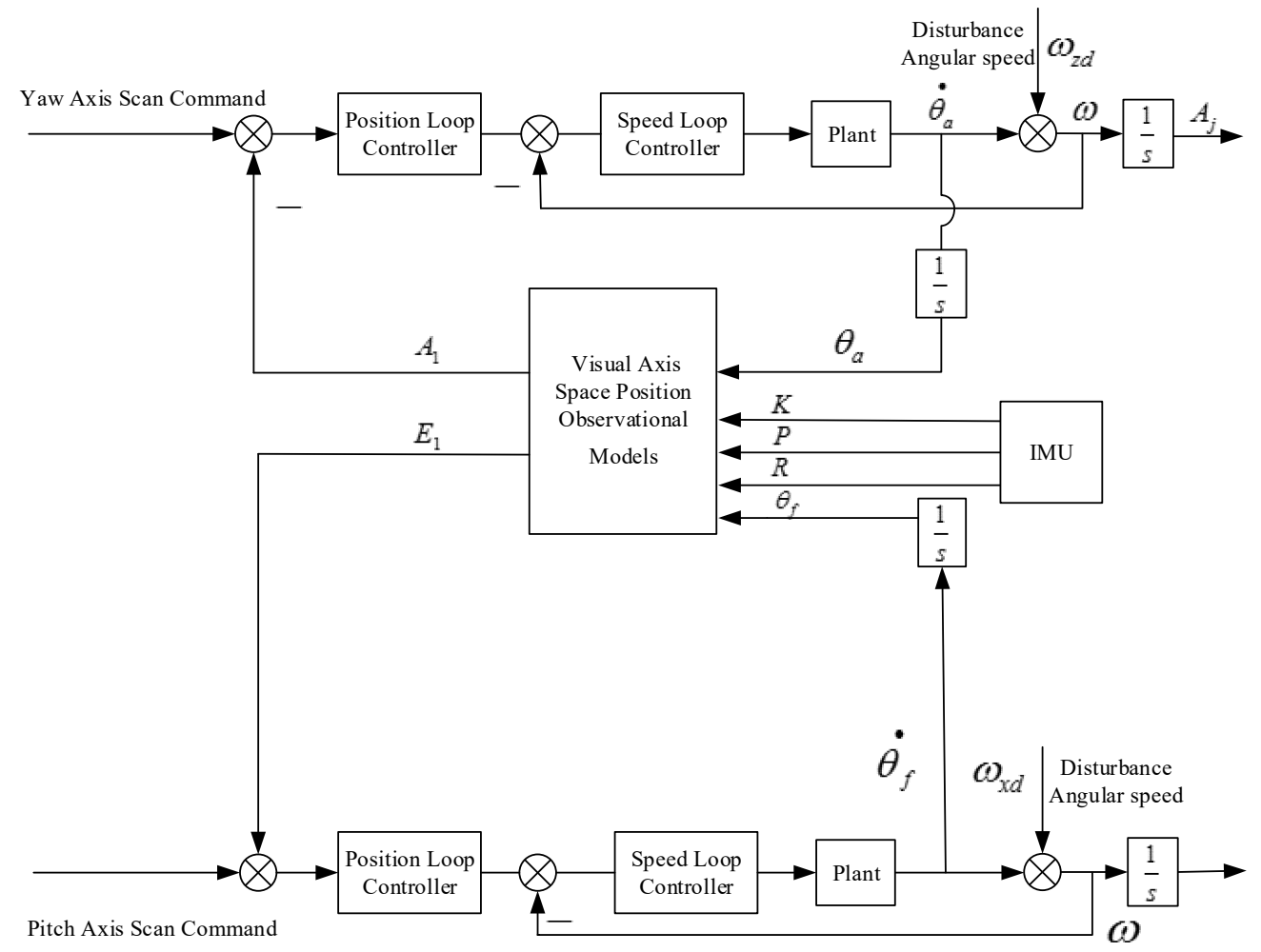

Fig. 8 Coordinate Transformation Relationship when Roll Angle Changes

The double closed-loop control principle not only isolates the disturbance angular velocity of the airplane, but also guarantees the pointing accuracy of visual axis, so we can satisfies the rapidity and 
stability of the system response at the same time. The system control model in scan mode is shown in Figure 8.

Now we use SIMULINK to simulate scanning command. Assume that scanning command of the yaw axis is $A=100^{\circ} \sin (2 \pi 0.1 t)$ and the pitch axis is $E=5^{\circ} \sin (2 \pi t)$. At the same time, the airplane disturbance angular velocity is $\omega_{x d}=1^{\circ} \cos (2 \pi t), \omega_{y d}=1^{\circ} \cos (2 \pi t), \omega_{z d}=$ $10^{\circ} \cos (2 \pi 0.1 t)$. We obtain the following the visual axis position observation in the yaw and pitch axis. The actual spatial position of the yaw axis is stabilized within $\pm 0.0004^{\circ}$, and the actual spatial position of the pitch axis is stable within $\pm 0.0007^{\circ}$.
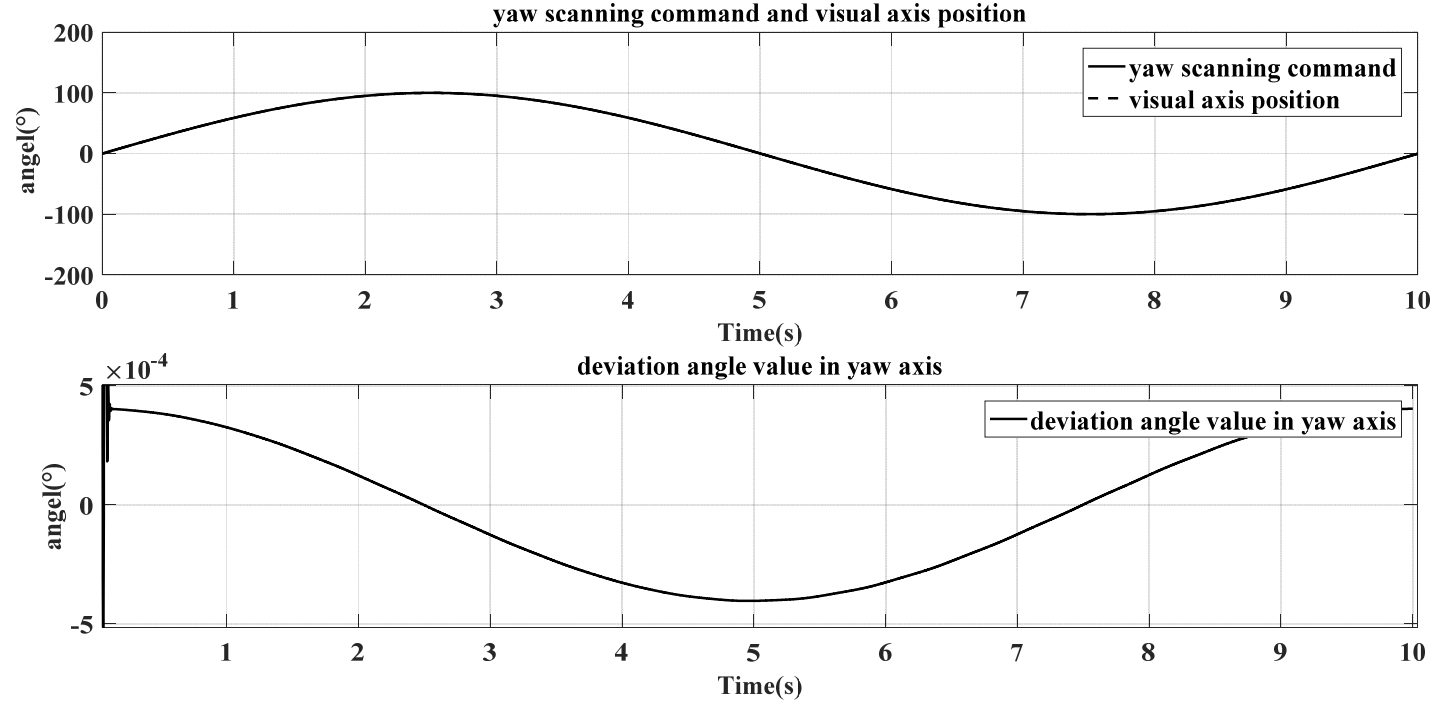

Fig. 9 Visual Axis Scanning Command and Actual Position in Yaw Axis
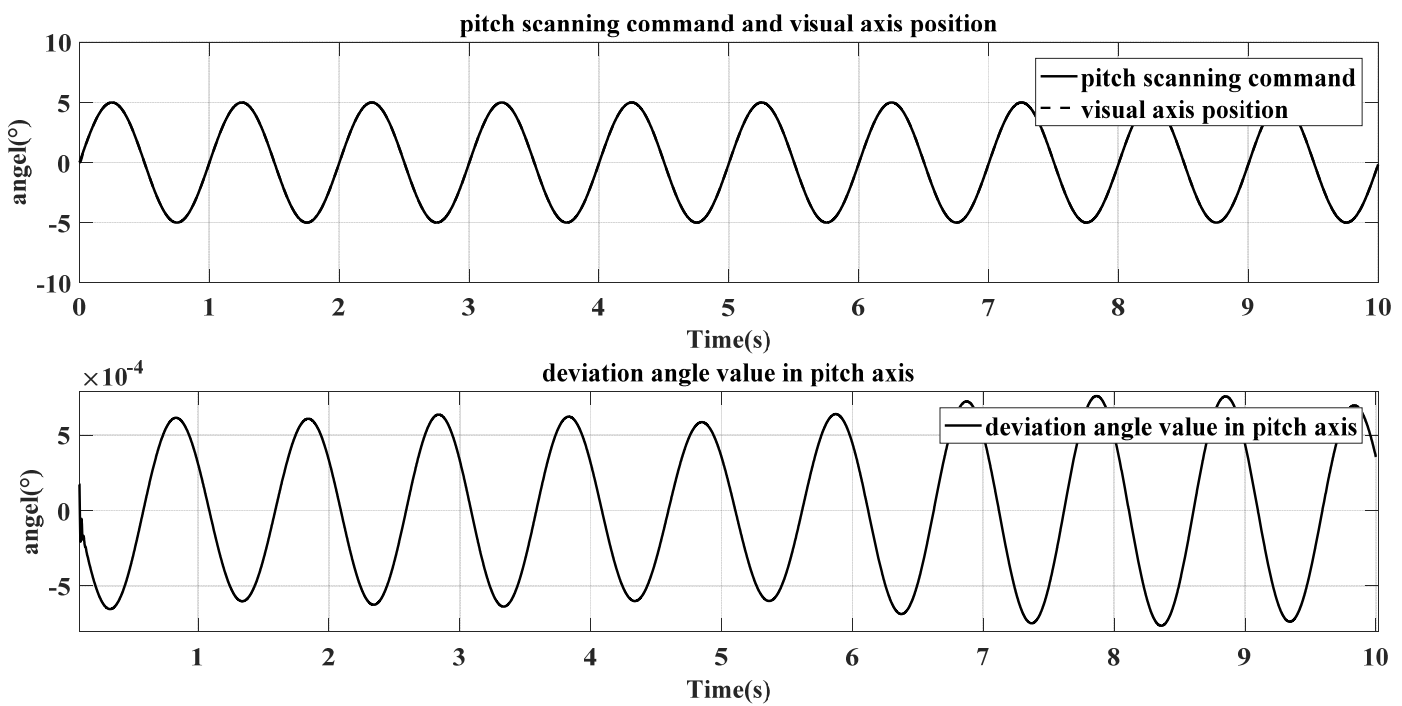

Fig. 10 Visual Axis Scanning Command and Actual Position in Pitch Axis

\section{Summary}

This paper first introduces the overall structure design of an airborne photoelectric tracking system. Then we establish a compensation model for the yaw and pitch two-axis airplane disturbances and realizes the inertial velocity compensation in the tracking mode. Similarly, the control block diagram is given when system works in scanning mode, and the inertial space position observation model with two freedom degrees of yaw and pitch is established. Consequently, the closed-loop inertial space position of two visual axes is completed. Finally, we verify the visual axis stability control algorithm on the SIMULINK simulation model. We can satisfy the rapidity and stability of the system response at the same time. 


\section{References}

[1]. Qiang Liu. Survey on Motion Control Technologies of High Performance Mechanical Servo Systems. ELECTRIC MACHINES AND CONTROL, Vol. 12 (2008) No. 5, p. 603-609.

[2]. Lizhi Sun. Research on Line of Sight Stabilization of Electro-Optical Stabilized Platform. (Master of Engineering, Harbin Institute of Technology, China 2017). p. 12-17.

[3]. Dongyang, Zhang Qinghe, Wu Xiaolan, et al. Design and Implementation of Active Disturbance Rejection Control for the Ship-borne Photoelectric Tracking Servo System. Control \& Decision Conference. Chongqing, China, 2017, p. 3951-3956.

[4]. Hongjie Hu, Teng Hou, Dapeng Lin. Design and Implementation of PMSM Control System Based on DSP and FPGA. Computer Measurement \& Control, Vol. 23(2015) No. 11, p. 36543656.

[5]. Xiang X, Li W, Liu Q, et al. Model and control for inner loop of electro-optical tracking servo system. IEEE International Conference on Mechatronics and Automation. IEEE. Tianjin, China, 2014, p. 1091-1095.

[6]. Tianya Zheng. Research on Control Method for Gyro-based Stabilization of Shipboard ElectroOptical Theodolite (Master of Engineering, Chinese Academy of Science, China 2016). p. 42-48.

[7]. Xuanlin Huang, Sisi Chen, Tao Tang. Research on Photoelectric Tracking System Based on Rotating Voice Coil Motor. Global Conference on Mechanics and Civil Engineering, Guangzhou, China, 2017-06-24, p. 63-66.

[8]. Zheng J. Design of high precision photoelectric tracking system for shipborne video. Ship Science \& Technology, Vol. 40(2018) No. 3A, p. 133-135. 